\title{
On asymptotic periodicity of kernel double Markovian operators
}

\author{
Wojciech Bartoszek ${ }^{1}\left[\right.$ - Michał Krzemiński ${ }^{1}$
}

Received: 6 December 2019 / Accepted: 7 April 2020 / Published online: 16 April 2020

(c) The Author(s) 2020

\begin{abstract}
It is proved that a kernel, doubly Markovian operator $T$ is asymptotically periodic if and only if its deterministic $\sigma$-field $\Sigma_{d}(T)$ (equivalently $\Sigma_{d}\left(T^{*}\right)$ ) is finite. It follows that kernel doubly Markovian operator $T$ is asymptotically periodic if and only if $T^{*}$ is asymptotically periodic.
\end{abstract}

Keywords Constrictive Markov operator · Smoothing Markov operator ·

Deterministic $\sigma$-algebra

Mathematics Subject Classification 47A35 - 37A30 $\cdot$ 60J05

\section{Introduction}

Stochastic Markov processes may be described, defined and studied with the use of different mathematical theories. Depending on needs and context we may introduce them as a family of random variables (elements) $\left\{\xi_{t}\right\}_{t \in \Theta}$, defined on a fixed probability space and satisfying the so-called Markov property, or by the system of transition probabilities $\{P(x, A): x \in S, A \in \mathcal{G}\}$, where $(S, \mathcal{G})$ is a fixed measurable space (called a phase space). In another approach, one can entirely use functional analysis language and say that a Markov process is exactly a linear, positive operator $T$ (called Markov or stochastic) defined on some Banach function space $\mathfrak{X}\left(=L^{p}\right.$ or $\left.C(S)\right)$, which satisfies other additional properties (for instance $T \mathbf{1}=\mathbf{1}$ if $\mathbf{1} \in \mathfrak{X}$ ). The methods and techniques of functional analysis are commonly accepted in a noncommutative Markov processes theory.

Wojciech Bartoszek

bartkowk@pg.edu.pl

Michał Krzemiński

mickrzem@pg.edu.pl

1 Department of Probability and Biomathematics, Gdańsk University of Technology, ul. Narutowicza 11/12, 80233 Gdańsk, Poland 
The evolution and asymptotic properties of a Markov process $\left\{\xi_{t}\right\}_{t \in \Theta}$ (once it is carried over to the operator theory framework) is reflected as an asymptotic behaviour (limit) of iterates $T^{n}$. There are many monographs on Markov processes, where probability theory and functional analysis intertwine. We shall borrow ideas, notions and inspiration from [8], which still may serve as a very good source of knowledge on limits of Markov processes (mainly for discrete time). Some other monographs $[4,6,18,19,21,23]$ were used once working on this project.

We revisit the notion of smoothness (smoothing) defined as some property of trajectories $T^{n} f$, where $f \in L^{1}(\mu)$ are densities (i.e. $f \geq 0$ and $\int f d \mu=1$ ). The general idea of smoothing says that as long as integrals $\int_{E} T^{n} f d \mu$ do not go uncontrolled on small sets $E$, the asymptotic behavior of $T^{n}$ becomes periodic. The notion of smoothness may be attributed to A. Lasota (cf. $[14,16,17]$ ), who jointly with collaborators established many interesting results concerning limit properties of Markov processes (Markov, stochastic operators) under smoothing assumptions.

This paper has been ignited by Y. Iwata (see [10]), who considered constrictivity and smoothness for the class of Markovian operators possessing a strictly positive stationary density. His paper is focused entirely on kernel Markov operators. Here we give some commentaries on Iwata work and generalize his main result. Finishing this section it is perhaps worth to mention that besides Lasota's seminal papers [16,17] on constrictivity, essential contribution to this topic was added by Komornik, Li, Yorke, Sine, Emel'yanov, Wolff, Miklavčič and Bartoszek (cf. [1,2,5,7,11-14,17,20,22]).

\section{Preliminaries and notation}

Let $(\mathfrak{X},\|\cdot\|)$ be a (real) Banach space. A linear operator $T: \mathfrak{X} \rightarrow \mathfrak{X}$ (in this paper we deal with contractions $\|T\| \leq 1$ ) is called constrictive if there exists a norm compact set $\mathcal{K} \subset \mathfrak{X}$ such that for every $\|x\| \leq 1$ the trajectory $T^{n} x$ is attracted to $\mathcal{K}$ (i.e. $\lim _{n \rightarrow \infty} \operatorname{dist}\left(T^{n} x, \mathcal{K}\right)=0$ ). If $\mathcal{K}$ is weakly compact and $\lim _{n \rightarrow \infty} \operatorname{dist}\left(T^{n} x, \mathcal{K}\right)=0$, for all $x$ from the unit ball of $\mathfrak{X}$, then $T$ is said to be weakly constrictive. After Lasota, $\mathcal{K}$ is called constrictor (or weak constrictor, respectively).

Definition 1 A linear operator $T: \mathfrak{X} \rightarrow \mathfrak{X}$ is called asymptotically periodic if there exist a system of vectors $g_{1}, \ldots, g_{r} \in \mathfrak{X}, \Lambda_{1}, \ldots, \Lambda_{r} \in \mathfrak{X}^{*}$ and a permutation $\alpha$ of the set of indices $\{1, \ldots, r\}$, such that

$$
\lim _{n \rightarrow \infty}\left\|T^{n} x-\sum_{j=1}^{r} \Lambda_{j}(x) g_{\alpha^{n}(j)}\right\|=0
$$

for all $x \in \mathfrak{X}$. If $r=1$, i.e. $\lim _{n \rightarrow \infty}\left\|T^{n} x-\Lambda(x) g\right\|=0$ for all $x \in \mathfrak{X}$, then we say that $T$ is asymptotically stable.

Let $(X, \mathcal{F}, \mu)$ be a $\sigma$-finite measure space and $\mathfrak{X}=L^{p}(\mu)$ stand for the classical Banach lattice of real valued measurable functions satisfying $\int_{X}|f|^{p} d \mu<\infty$ with the ordinary norm $\|\cdot\|_{p}$ (functions equal $\mu$ almost everywhere are identified). A linear operator $T: L^{1}(\mu) \rightarrow L^{1}(\mu)$ is called Markov if $T f \geq 0$ and $\int_{X} T f d \mu=\int_{X} f d \mu$ 
for all nonnegative $f \in L_{+}^{1}(\mu)$. In other words, $T$ is Markov if $T \geq 0$ and $T^{*} \mathbf{1}=\mathbf{1}$, where $T^{*}: L^{\infty}(\mu) \rightarrow L^{\infty}(\mu)$. Clearly, the operator norm $\|T\|=1$, so Markov operators are contractions. $f \in L^{1}(\mu)$ is called a density if $f \geq 0$ and $\int_{X} f d \mu=1$. The closed convex set of all densities in $L^{1}(\mu)$ is denoted by $\mathcal{D}$.

It was proved in [17] (Theorem 1.1) that, if $T$ is a constrictive Markov operator on $L^{1}(\mu)$, then $T$ is asymptotically periodic. This result was subsequently extended on $L^{p}(\mu)$ spaces, general Banach lattices and recently on ordered Banach spaces with bases (cf. $[1,5,20,22])$. We do not aim to dive into abstract regions but focus on classical spaces $\mathfrak{X}=L^{1}(\mu)$. We notice that if there exists an invariant (stationary) density $T f_{*}=f_{*}$, which is strictly positive $\mu$ a.e., then we may introduce $\widetilde{T} f=$ $T\left(f f_{*}\right) / f_{*}$, which is defined on $L^{1}\left(f_{*} \mu\right)$. Clearly for all $f \in L^{1}\left(f_{*} \mu\right)$ we have $\widetilde{T}^{n} f=T^{n}\left(f f_{*}\right) / f_{*}$, so the dynamics of $T^{n}$ may be deduced from $\widetilde{T}^{n}$. The Markov operator $\widetilde{T}$ satisfies $\widetilde{T} \mathbf{1}=\mathbf{1}$, where $\mathbf{1}$ is simply a density function on the probability measure space $\left(X, \mathcal{F}, f_{*} \mu\right)$.

Therefore, without loss of generality, we shall assume throughout this paper that $(X, \mathcal{F}, \mu)$ is a probability measure space and $\mathbf{1} \in L^{1}(\mu)$ is a stationary density of a Markov operator $T: L^{1}(\mu) \rightarrow L^{1}(\mu)$. Such Markov operators $T$ are called double Markovian (double stochastic), as $T^{*}: L^{\infty}(\mu) \rightarrow L^{\infty}(\mu)$ may be extended to $L^{1}(\mu) \supseteq L^{\infty}(\mu)$ and $T^{*}$ becomes again a Markov operator. Simply both $T$ and $T^{*}$ are Markovian linear operators acting simultaneously on $L^{1}(\mu)$ and $L^{\infty}(\mu)$ as positive linear contractions. Notice that $T^{* *}=T$ (see [4], Proposition 1.1).

We recall an introduced by Lasota and Komornik notion of smoothness.

Definition 2 We say that a Markovian operator $T: L^{1}(\mu) \rightarrow L^{1}(\mu)$ is smoothing if there exist constants $0<\eta<1$ and $\delta>0$ such that for every density $f \in \mathcal{D}$ there exists a natural $n_{f}$, such that for all $n \geq n_{f}$ we have

$$
\int_{E} T^{n} f d \mu \leq \eta, \text { for all } E \in \mathcal{F} \text { satisfying } \mu(E) \leq \delta .
$$

Asymptotic periodicity, smoothness and constrictivity of Markovian operators on $L^{1}(\mu)$ appear to be closely related through the Komornik and Lasota works (see [1114]). Gathering their results we arrive to the following characterization.

Theorem 1 Let $(X, \mathcal{F}, \mu)$ be a probability space and $T: L^{1}(\mu) \rightarrow L^{1}(\mu)$ be a Markov operator. Then the following are equivalent:

(1) $T$ is asymptotically periodic,

(2) $T$ is constrictive,

(3) $T$ is weakly constrictive,

(4) $T$ is smoothing.

In [10] the author studied conditions for smoothness (constrictivity) of doubly Markovian and kernel operators. He borrowed most of his ideas and techniques from the Foguel's monograph (chapters V and VIII). However the power of [8] has not been exploited to its limits and potential. Let us start recalling necessary notation from [8].

A Markov operator $T: L^{1}(\mu) \rightarrow L^{1}(\mu)$ is said to be conservative if $\sum_{n=0}^{\infty} T^{n} f(x)=+\infty \quad \mu$ a.e. for some (equivalently for all) strictly positive 
$f \in L^{1}(\mu)$. If $T$ is conservative and $T^{*} h \leq h$ for some $h \in L^{\infty}(\mu)$ then $T^{*} h=h$. Clearly, each Markov operator with strictly positive stationary density is conservative. In particular, doubly Markovian operators are conservative. A conservative operator $T$ is called ergodic (totally ergodic), if $T^{*} h=h\left(T^{* k} h=h\right.$ for some $\left.k \geq 1\right)$ implies that $h=$ const $\mu$ a.e. where $h \in L^{\infty}(\mu)$. Ergodicity means that $T^{*} \mathbf{1}_{A}=\mathbf{1}_{A}$ holds only when $\mu(A)=0$ or $\mu(A)=1$. Let us recall that conservative Markov operators $T$ (in particular all doubly Markovian operators) are non-disappearing, i.e. if $T^{*} f=0$ for some $f \geq 0$, then $f=0$. Hence, (see Lemma 0 in [15] for all details) if $T^{*} g=\mathbf{1}_{A}$ with $0 \leq g \leq 1$, then there exists a unique $E \in \mathcal{F}$ such that $g=\mathbf{1}_{E}$. The family of all $A \in \mathcal{F}$, such that for every $n$ there exists $A_{n} \in \mathcal{F}$, such that $T^{* n} \mathbf{1}_{A}=\mathbf{1}_{A_{n}}$, is denoted by $\Sigma_{\mathrm{d}}(T)$. $\Sigma_{\mathrm{d}}(T)$ is a sub $\sigma$-algebra if $T$ is double Markovian, and it is called a deterministic $\sigma$-algebra. By $\Sigma_{1}(T)$ we denote a sub $\sigma$-algebra of $\Sigma_{\mathrm{d}}(T)$ of all those sets $A \in \mathcal{F}$ such that for every natural $n$ we have $T^{* n} T^{n} \mathbf{1}_{A}=T^{n} T^{* n} \mathbf{1}_{A}=\mathbf{1}_{A}$. By the symmetry $\Sigma_{1}(T)=\Sigma_{1}\left(T^{*}\right)$.

Given a doubly Markovian operator $T$ consider supports $B_{f}=\{x \in X: f(x)>0\}$ of a nonnegative $f \in L^{1}(\mu)$ and $B_{T f}=\{x \in X: T f(x)>0\}$ corresponding to its image $T f$. We notice that

$$
\begin{aligned}
\|f\|_{1} & =\|T f\|_{1}=\int_{B_{T f}} T f d \mu=\int_{X} f \cdot\left(T^{*} \mathbf{1}_{B_{T f}}\right) d \mu \\
& =\int_{B_{f}} f \cdot\left(T^{*} \mathbf{1}_{B_{T f}}\right) \leq \int_{B_{f}} f d \mu=\|f\|_{1} .
\end{aligned}
$$

Hence $T^{*} \mathbf{1}_{B_{T f}} \geq \mathbf{1}_{B_{f}}$. We obtain $\mu\left(B_{T f}\right) \geq \mu\left(B_{f}\right)$ as $T^{*}$ is also Markovian.

We say that a Markov operator $T$ is regular, if there exists a family $\{P(x, \cdot)\}_{x \in X}$ of probability measures $P(x, \cdot)$ on $(X, \mathcal{F})$, such that for every $A \in \mathcal{F}$ the mapping $X \ni x \rightarrow P(x, A)$ is $\mathcal{F}$ measurable, and

$$
T^{*} h(x)=\int_{X} h(y) P(x, d y), \quad \int_{A} T f(x) d \mu(x)=\int_{X} f(x) P(x, A) d \mu(x)
$$

for all $f \in L^{1}(\mu), A \in \mathcal{F}$ and $h \in L^{\infty}(\mu)$.

A double Markovian operator $T$ is called integral (or kernel) if it has an integral representation $T f(y)=\int_{X} k(x, y) f(x) d \mu(x)$, where $k: X \times X \rightarrow R_{+}$is a jointly measurable function satisfying $\int_{X} k(x, y) d \mu(y)=1$ for $\mu$ almost all $x$ and $\int_{X} k(x, y) d \mu(x)=1$ for $\mu$ almost all $y$. Clearly, all kernel Markov operators (see $[9,23])$ are regular and $P(x, A)=\int_{X} k(x, y) \mathbf{1}_{A}(y) d \mu(y)$.

It follows that kernel, double Markovian operators are conservative and therefore Harris operators. In this situation $\Sigma_{d}(T)$ is atomic (the geometric structure of Harris operators and their analytical properties are well known, and are comprehensively presented in [8], Theorem D, p. 58).

\section{Result}

The main goal of this note is to upgrade results of [10] (see below). 
Theorem 2 ([10]) Let $T: L^{1}(\mu) \rightarrow L^{1}(\mu)$ be a kernel double Markovian operator. Then the following are equivalent:

(1) $T$ is smoothing (asymptotically periodic),

(2) the deterministic sub $\sigma$-algebra $\Sigma_{d}\left(T^{*}\right)$ has at most finitely many atoms and if $q$ denotes the least common multiple of orders of atoms in $\Sigma_{d}\left(T^{*}\right)$ then for every atom $W \in \Sigma_{d}\left(T^{*}\right)$ one has

$$
\lim _{n \rightarrow \infty} \mu\left(A \backslash \operatorname{supp}\left(T^{n q} \mathbf{1}_{B}\right)\right)=0
$$

for all measurable $A, B \subseteq W$.

We will generalize the above mentioned theorem and shall prove that for kernel double Markovian operator $T$, in condition (2), finiteness of $\Sigma_{d}\left(T^{*}\right)$ is sufficient and no extra verifications like $\lim _{n \rightarrow \infty} \mu\left(A \backslash \operatorname{supp}\left(T^{n q} \mathbf{1}_{B}\right)\right)=0$ for all measurable $A, B \subseteq W$, need to be done. Definitely, this will bring the Iwata result to its refined and final shape. Let us mention that, looking from the point of view of structure of $\Sigma_{d}\left(T^{*}\right)$, asymptotic behaviour of iterates $T^{n} f$ was studied in [3]. Finiteness of $\Sigma_{d}\left(T^{*}\right)$ was guaranteed by assuming that $T$ almost overlaps supports (see [3], Proposition 1), which was additionally supported with another assumption that trajectories $T^{n} f$ have strong limit points. However, doubly Markovian operators $T$ were not necessarily kernel. Here we show that for kernel and doubly Markovian $T$, we can (if we wish) replace $\Sigma_{d}\left(T^{*}\right)$ for a (classical) deterministic $\sigma$-field $\Sigma_{d}(T)$. For this let us start with the following lemma, which is proved with all details, even though a specialist on ergodic theory of Markov operators may relatively easily extract its proof from [8].

Lemma 1 Let $T: L^{1}(\mu) \rightarrow L^{1}(\mu)$ be a double Markovian operator. If $\Sigma_{d}(T)$ is finite then $\Sigma_{d}(T)=\Sigma_{1}(T) \subseteq \Sigma_{d}\left(T^{*}\right)$.

Proof We start from a general observation: if $T^{*} \mathbf{1}_{E}=\mathbf{1}_{F}$ then $\mu(E)=\mu(F)$, and $\mu(E) \geq \int_{X} \mathbf{1}_{E} T \mathbf{1}_{F} d \mu=\int_{X} T^{*} \mathbf{1}_{E} \mathbf{1}_{F} d \mu=\int_{X} \mathbf{1}_{F} \mathbf{1}_{F} d \mu=\mu(F)=\mu(E)$. Hence, $T \mathbf{1}_{F}=\mathbf{1}_{E}$. It follows from ([15], Lemma 0) that if $T^{*} \mathbf{1}_{E_{1}}=T^{*} \mathbf{1}_{E_{2}}=\mathbf{1}_{F}$, then $E_{1}=E_{2}$. Thus, $T^{*} \mathbf{1}_{E}=\mathbf{1}_{F}$ if and only $T \mathbf{1}_{F}=\mathbf{1}_{E}$.

Let $\mathcal{A}=\left\{A_{1}, \ldots, A_{a}\right\}$ be the family of all atoms of $\Sigma_{d}(T)$. We partition $\mathcal{A}$ into subfamilies $\mathcal{A}_{k}$, such that $\mu(A)=\gamma_{k}$ for all $A \in \mathcal{A}_{k}$, where $\gamma_{1}<\gamma_{2}<\cdots<\gamma_{m}$. Let $A_{l} \in \mathcal{A}_{1}$ be arbitrary. We have $T^{*} \mathbf{1}_{A_{l}}=\mathbf{1}_{A_{\beta(l)}}$, where $A_{\beta(l)} \in \Sigma_{d}(T)$. Clearly, $A_{\beta(l)} \in \mathcal{A}_{1}$ is an atom. The transformation $A_{l} \rightarrow A_{\beta(l)}$ on the set $\mathcal{A}_{1}$ is a bijection (we do not claim that $\beta$ is one cycle).

Then considering $T^{*} \mathbf{1}_{A_{l}}=\mathbf{1}_{A_{\beta(l)}}$ on $\mathcal{A}_{2}$ we obtain that again $A_{l} \rightarrow A_{\beta(l)}$, on the set $\mathcal{A}_{2}$, is a bijection. We continue with these arguments to exhaust the whole partition $\mathcal{A}$.

Because $\Sigma_{d}(T)$ is finite $T^{*}: \operatorname{span}\left(\left\{\mathbf{1}_{A_{1}}, \ldots, \mathbf{1}_{A_{a}}\right\}\right) \rightarrow \operatorname{span}\left(\left\{\mathbf{1}_{A_{1}}, \ldots, \mathbf{1}_{A_{a}}\right\}\right)$ is linear, positive and invertible with $T^{*^{-1}}=T$. Hence, for any atom $A_{l} \in \Sigma_{d}(T)$, and any natural $n$, we have $T^{n} \mathbf{1}_{A_{l}}=\mathbf{1}_{A_{\alpha^{n}(l)}}$, where $\alpha=\beta^{-1}$ is a permutation of the set $\{1,2, \ldots, a\}$. It follows that $T^{n} T^{* n} \mathbf{1}_{A}=T^{* n} T^{n} \mathbf{1}_{A}=\mathbf{1}_{A}$ for all $A \in \mathcal{A}$. By linearity $T^{n} T^{* n} \mathbf{1}_{A}=T^{* n} T^{n} \mathbf{1}_{A}=\mathbf{1}_{A}$ for all $A \in \Sigma_{d}(T)$, as $\mathbf{1}_{A}$ has a unique representation as a sum $\mathbf{1}_{A}=\sum_{j \in J} \mathbf{1}_{A_{j}}$, where $J \subseteq\{1,2, \ldots, a\}$ and $A_{j} \in \mathcal{A}$. We have obtained $\Sigma_{d}(T)=\Sigma_{1}(T) \subseteq \Sigma_{d}\left(T^{*}\right)$. 
The following corollary is a direct consequence of the above Lemma 1.

Corollary 1 Let $T: L^{1}(\mu) \rightarrow L^{1}(\mu)$ be a double Markovian operator. If $\sigma$-algebras $\Sigma_{d}(T)$ and $\Sigma_{d}\left(T^{*}\right)$ are finite then $\Sigma_{d}(T)=\Sigma_{1}(T)=\Sigma_{d}\left(T^{*}\right)$.

Now we are ready to explain mutual relations between deterministic $\sigma$-fields $\Sigma_{d}(T)$ and $\sigma_{d}\left(T^{*}\right)$ if $T$ is kernel.

Theorem 3 Let $T$ be a kernel doubly Markovian operator. The deterministic $\sigma$-algebra $\Sigma_{d}(T)$ (equivalently $\Sigma_{d}\left(T^{*}\right)$ ) is finite if and only if $\Sigma_{d}\left(T^{*}\right)$ is finite (respectively $\Sigma_{d}(T)$ is finite) and then $\Sigma_{d}(T)=\Sigma_{1}(T)=\Sigma_{d}\left(T^{*}\right)$.

Proof The $\sigma$-algebras $\Sigma_{d}(T)$ and $\Sigma_{d}\left(T^{*}\right)$ are atomic as $T$ and $T^{*}$ are kernel and conservative. By Lemma 1 we have $\Sigma_{d}(T)=\Sigma_{1}(T) \subseteq \Sigma_{d}\left(T^{*}\right)$. Denote $\left\{W_{1}, \ldots, W_{a}\right\}$ to be the family of all atoms of $\Sigma_{d}(T)$. We choose and fix for a while an atom $W_{i}=W$. Let $r_{i}=r$ be the period of $W$ (i.e. $r$ is the least $j \in \mathbb{N}_{0}$ such that $T^{* j} \mathbf{1}_{W}=\mathbf{1}_{W}$ ). We introduce locally $S: L^{1}\left(W, \mathcal{F}_{W}, \mu_{W}\right) \rightarrow L^{1}\left(W, \mathcal{F}_{W}, \mu_{W}\right)$ defined as $S=T^{r}$. Here $\mathcal{F}_{W}=\{E \cap W: E \in \mathcal{F}\}$ denotes the trace $\sigma$-algebra and $\mu_{W}(E)=\frac{\mu(E)}{\mu(W)}$ is the conditional probability measure. Clearly, $S$ is a kernel doubly Markovian operator on $L^{1}\left(\mu_{W}\right)$. In particular, it is conservative and non-disappearing (and of course kernel).

Similarly if $E \in \mathcal{F}_{W}$ belongs to $\Sigma_{d}(S)$, then $S^{* k} \mathbf{1}_{E}=T^{* r k} \mathbf{1}_{E}=\mathbf{1}_{F_{r k}}$, for some $F_{r k}=E_{k} \in \mathcal{F}_{W}$. Again by [15] we have $T^{* j} \mathbf{1}_{E}=\mathbf{1}_{F_{j}}$ for all $0 \leq j \leq r k$. In other words $\Sigma_{d}(S) \subseteq \Sigma_{d}(T)$. But $W \in \Sigma_{d}(T)$ is taken to be an atom, so $E=W$. Thus, $\Sigma_{d}(S)=\{\emptyset, W\}$ is trivial.

Let us suppose that $E \in \Sigma_{d}\left(S^{*}\right)$, for some $0<\mu_{W}(E)<1$. Hence, $S^{k} \mathbf{1}_{E}=\mathbf{1}_{E_{k}}$ for all $k \in \mathbb{N}$, where $E_{k} \in \mathcal{F}_{W}$ satisfy $\mu_{W}\left(E_{k}\right)=\mu_{W}(E)$. It is well known that kernel doubly Markovian operators transform order intervals $[0, f] \subseteq L^{1}$ into norm $\|\cdot\|_{1}$ relatively compact subsets (see [21], Proposition IV.9.8). Thus we may find an increasing subsequence $k_{j} \rightarrow \infty$ such that $\lim _{j \rightarrow \infty}\left\|S^{k_{j}} \mathbf{1}_{E}-g\right\|_{1}=0$, where $0 \leq g \leq \mathbf{1}_{W}$. Actually $g=\mathbf{1}_{F}$ for some $F \in \mathcal{F}_{W}$ ( $g$ may be approximated by characteristic functions as close as we wish). Clearly $0<\mu_{W}(F)=\mu_{W}(E)<1$. For an arbitrary $i \in \mathbb{N}$ let us consider $S^{* i} \mathbf{1}_{F}=\lim _{j \rightarrow \infty} S^{* i} S^{k_{j}} \mathbf{1}_{E}=\lim _{j \rightarrow \infty} S^{k_{j}-i} \mathbf{1}_{E}=$ $\mathbf{1}_{F_{i}}$ for some $F_{i} \in \mathcal{F}_{W}$ (if necessary we may choose another subsequence from $k_{j}-i$ and again apply the compactness argument). Hence, $F \in \Sigma_{d}(S)$, a contradiction as $W$ is an atom of $\Sigma_{d}(S)$. Thus, $\Sigma_{d}\left(S^{*}\right)=\{\emptyset, W\}$ is trivial.

Applying these considerations to all (finitely many) atoms $W_{j} \in \Sigma_{d}(T)$ we obtain that each atom $F \in \Sigma_{d}\left(T^{*}\right)$ has a representation $F=\bigcup_{j=1}^{a} F \cap W_{j} \in \Sigma_{d}(T)$ as $F \cap W_{j}$ is $W_{j} \in \Sigma_{d}(T)$ or $\emptyset$. Hence, $\Sigma_{d}\left(T^{*}\right) \subseteq \Sigma_{d}(T)=\Sigma_{1}(T) \subseteq \Sigma_{d}\left(T^{*}\right)$ and all are finite.

Remark 1 In the above Theorem 3 the assumption that $T$ is kernel is essential. In fact, consider the Frobenius-Perron operator associated with the transformation $\tau(x)=$ (2x) $\bmod 1$. It is commonly known that $\tau$ preserves the Lebesgue measure $\lambda$ on $X=[0,1)$. In particular, $T$ is doubly Markovian. Clearly $\Sigma_{d}(T)=\mathcal{B}_{[0,1)}$ is the full $\sigma$ algebra (simply $T^{*} h(x)=h(\tau(x))$, where $h \in L^{\infty}(\lambda)$, is the composition operator). On the other hand $\tau$ is exact ( $T$ is asymptotically stable), so $\lim _{n \rightarrow \infty} T^{n} \mathbf{1}_{A}=\lambda(A) \mathbf{1}$ in the $L^{1}$ norm (cf. [18]). Hence,

$$
\Sigma_{d}\left(T^{*}\right)=\left\{A \in \mathcal{B}_{[0,1)}: T^{j} \mathbf{1}_{A}=\mathbf{1}_{A_{j}} \text { for all } j=0,1,2, \ldots\right\}=\{\emptyset,[0,1)\}
$$


is the trivial $\sigma$-algebra, thus finite. Nevertheless $\Sigma_{d}(T) \neq \Sigma_{d}\left(T^{*}\right)$.

By [8] (Theorem A, p. 85) for any $f \in L^{2}(\mu)$ such that $\int_{X} f h d \mu=0$ for all $h \in L^{2}\left(X, \Sigma_{1}(T), \mu\right)$ we have $\lim _{n \rightarrow \infty} T^{n} f=0$ weakly. If $T$ is kernel we have even strong convergence $\lim _{n \rightarrow \infty}\left\|T^{n} f\right\|_{2}=0$ (again apply [21], Proposition IV.9.8). We may easily modify it to $\lim _{n \rightarrow \infty}\left\|T^{n} f\right\|_{1}=0$. Therefore, if $T$ is kernel, doubly Markovian with finite $\Sigma_{d}(T)=\Sigma_{d}\left(T^{*}\right)=\Sigma_{1}(T)$ and $W$ is an atom of period $r$, then $L^{2}\left(W, \Sigma_{1}(S), \mu_{W}\right)=\left\{t \mathbf{1}_{W}: t \in \mathbb{R}\right\}$. Thus, for all $f$ satisfying $\int_{W} f d \mu_{W}=0$ we have $\lim _{n \rightarrow \infty}\left\|S^{n} f\right\|_{1}=0$ and $\lim _{n \rightarrow \infty}\left\|S^{* n} f\right\|_{1}=0$. Notice that $\int_{W}[f-$ $\left.\left(\int_{W} f d \mu\right) \mathbf{1}_{W}\right] d \mu_{W}=0$ for all $f \in L^{1}(\mu)$. We have obtained:

Corollary 2 Let $T: L^{1}(\mu) \rightarrow L^{1}(\mu)$ be a kernel double Markovian operator. If $\Sigma_{d}(T)$ is finite (by Theorem 3 equivalently $\Sigma_{d}\left(T^{*}\right)$ is finite) then for each atom $W \in \Sigma_{d}(T)$ with period $r$ we have

$$
\lim _{n \rightarrow \infty}\left\|T^{r n} f-\frac{1}{\mu(W)}\left(\int_{W} f d \mu\right) \mathbf{1}_{W}\right\|_{1}=0,
$$

and by symmetry

$$
\lim _{n \rightarrow \infty}\left\|T^{* r n} f-\frac{1}{\mu(W)}\left(\int_{W} f d \mu\right) \mathbf{1}_{W}\right\|_{1}=0
$$

for all $f \in L^{1}(\mu)$ concentrated on $W$.

Moreover, denoting $\left\{A_{1}, \ldots, A_{a}\right\}$ to be the family of all atoms of $\Sigma_{d}(T)$, there exists (see our Lemma 1) a permutation $\alpha:\{1, \ldots, a\} \rightarrow\{1, \ldots, a\}$ such that $T \mathbf{1}_{A_{j}}=$ $\mathbf{1}_{A_{\alpha(j)}}$ and $T^{*} \mathbf{1}_{A_{j}}=\mathbf{1}_{A_{\alpha-1}(j)}$.

Now we are in a position to formulate the main result of the paper.

Theorem 4 Let $T: L^{1}(\mu) \rightarrow L^{1}(\mu)$ be a kernel doubly Markovian operator. Then the following are equivalent

(1) $\Sigma_{d}(T)\left(\right.$ or $\left.\Sigma_{d}\left(T^{*}\right)\right)$ is finite with atoms $\left\{A_{1}, A_{2}, \ldots, A_{a}\right\}$,

(2) $\Sigma_{d}(T)=\Sigma_{d}\left(T^{*}\right)=\Sigma_{1}(T)$ are finite,

(3) $T$ and $T^{*}$ are simultaneously asymptotically periodic with

$$
\lim _{n \rightarrow \infty}\left\|T^{n} f-\sum_{j=1}^{a}\left(\int_{A_{j}} f d \mu\right) \mathbf{1}_{A_{\alpha^{n}(j)}}\right\|_{1}=\lim _{n \rightarrow \infty}\left\|T^{* n} f-\sum_{j=1}^{a}\left(\int_{A_{j}} f d \mu\right) \mathbf{1}_{A_{\alpha^{-n}(j)}}\right\|_{1}=0,
$$

for all $f \in L^{1}(\mu)$, where the permutation $\alpha$ is described in our Lemma 1,

(4) $T$ is asymptotically periodic,

(5) $T$ is constrictive,

(6) $T$ is weakly constrictive,

(7) $T$ is smoothing. 
Proof The implication (1) $\Rightarrow$ (2) is proved in our Theorem 3, (3) $\Rightarrow$ (4) is obvious. The implications $(4) \Leftrightarrow(5) \Leftrightarrow \ldots \Leftrightarrow(7)$ may be recognized today as classical and hold for general (doubly) Markovian operators.

(2) $\Rightarrow$ (3) follows from Corollary 2. Let $A_{1}, A_{2}, \ldots, A_{a}$ be atoms of $\Sigma_{d}(T)=$ $\Sigma_{d}\left(T^{*}\right)$. For a fixed $f \in L^{1}(\mu)$ consider its partition $f=\sum_{j=1}^{a} \mathbf{1}_{A_{j}} f$. Let $q$ be the least common multiple of all (minimal) periods of atoms. Then

$$
\begin{aligned}
\lim _{n \rightarrow \infty} T^{q n} f & =\sum_{j=1}^{a} \lim _{n \rightarrow \infty} T^{q n}\left(\mathbf{1}_{A_{j}} f\right) \\
& =\sum_{j=1}^{a} \frac{1}{\mu\left(A_{j}\right)}\left(\int_{A_{j}} f d \mu\right) \mathbf{1}_{A_{j}},
\end{aligned}
$$

in the $L^{1}$ norm.

On limit functions the iterates behave periodically:

$$
T^{k}\left(\sum_{j=1}^{a}\left(\int_{A_{j}} f d \mu\right) \mathbf{1}_{A_{j}}\right)=\sum_{j=1}^{a}\left(\int_{A_{j}} f d \mu\right) \mathbf{1}_{A_{\alpha^{k}(j)}}
$$

and

$$
T^{* k}\left(\sum_{j=1}^{a}\left(\int_{A_{j}} f d \mu\right) \mathbf{1}_{A_{j}}\right)=\sum_{j=1}^{a}\left(\int_{A_{j}} f d \mu\right) \mathbf{1}_{A_{\alpha^{-k}(j)}} .
$$

The operators $T$ and $T^{*}$ are asymptotically periodic.

The proof of implication (7) $\Rightarrow$ (1) may be borrowed from [10]. For the sake of completeness of this paper we provide its proof (especially because we have replaced $\Sigma_{d}\left(T^{*}\right)$ for $\left.\Sigma_{d}(T)\right)$. Actually, we need only a fraction of Iwata's argument.

Let $\delta>0, \eta>0$ be as in Definition 2. Suppose that an atom $W \in \Sigma_{d}\left(T^{*}\right)$ satisfies $\mu(W) \leq \delta$. For all $n \geq 1$ we have $\mu\left(W_{n}\right)=\mu(W)$, where $T^{n} \mathbf{1}_{W}=\mathbf{1}_{W_{n}}$. Then $T^{* n} \mathbf{1}_{W_{n}}=\mathbf{1}_{W}$. Now let us consider the density $f=\frac{1}{\mu(W)} \mathbf{1}_{W}$. We have

$$
\int_{W_{n}} T^{n} f d \mu=\int_{X} f T^{* n} \mathbf{1}_{W_{n}} d \mu=\int_{X} f \mathbf{1}_{W} d \mu=1
$$

for all $n \in \mathbb{N}$, where $\mu\left(W_{n}\right) \leq \delta$. But the smoothness assumption requires $\int_{W_{n}} T^{n} f d \mu \leq \eta<1$ if $n$ is large enough, a contradiction. Hence $\mu(W) \geq \delta$ are separated from 0 for all atoms $W \in \Sigma_{d}\left(T^{*}\right)$. The $\sigma$-algebra $\Sigma_{d}\left(T^{*}\right)$ is finite, so $\Sigma_{d}(T)$ is also finite.

Corollary 3 Let $T: L^{1}(\mu) \mapsto L^{1}(\mu)$ be a kernel doubly Markovian operator. Then the following are equivalent 
(1) $T$ is asymptotically stable,

(2) $T^{*}$ is asymptotically stable (as a doubly Markovian operator on $L^{1}(\mu)$ ),

(3) $\Sigma_{d}(T)=\{\varnothing, X\}$,

(4) $\Sigma_{d}\left(T^{*}\right)=\{\emptyset, X\}$.

Open Access This article is licensed under a Creative Commons Attribution 4.0 International License, which permits use, sharing, adaptation, distribution and reproduction in any medium or format, as long as you give appropriate credit to the original author(s) and the source, provide a link to the Creative Commons licence, and indicate if changes were made. The images or other third party material in this article are included in the article's Creative Commons licence, unless indicated otherwise in a credit line to the material. If material is not included in the article's Creative Commons licence and your intended use is not permitted by statutory regulation or exceeds the permitted use, you will need to obtain permission directly from the copyright holder. To view a copy of this licence, visit http://creativecommons.org/licenses/by/4.0/.

\section{References}

1. Bartoszek, W.: Asymptotic periodicity of the iterates of positive contractions on Banach lattices. Stud. Math. 91, 179-188 (1988)

2. Bartoszek, W.: On uniformly smoothing stochastic operators. Comment. Math. Univ. Carol. 36(1), 203-206 (1995)

3. Bartoszek, W.: On asymptotic cyclicity of doubly stochastic operators. Ann. Polon. Math. 72(2), 145152 (1999)

4. Brown, J.R.: Ergodic Theory and Topological Dynamics. Academic Press, New York (1976)

5. Emel'yanov, E.Y.: Positive asymptotically regular operators in $L^{1}$ and KB-spaces. In: Proceedings of the Positivity IV-Theory and Applications Dresden (Germany), pp. 53-61 (2006)

6. Emel'yanov, E.Y.: Non-Spectral Asymptotic Analysis of One-Parameter Operator Semigroups, Operator Theory: Advances and Applications, vol. 173. Birkhäuser Verlag, Basel (2007)

7. Emel'yanov, E.Y., Wolff, M.: Quasi-constricted linear operators on Banach spaces. Stud. Math. 144(2), 169-179 (2001)

8. Foguel, S.R.: The Ergodic Theory of Markov Processes. Van Nostrand Reinhold, New York (1969)

9. Gerlach, M., Kunze, M.: On the lattice structure of kernel operators. Math. Nachr. 288(5-6), 584-592 (2015)

10. Iwata, Y.: Constrictive Markov operators induced by Markov processes. Positivity 20(2), 355-367 (2016)

11. Komornik, J.: Asymptotic periodicity of the iterates of Markov operators. Tôhoku Mat. J. 38, 15-27 (1986)

12. Komornik, J.: Asymptotic decomposition of smoothing positive operators. Acta Univ. Carol. 30(2), 77-81 (1989)

13. Komornik, J.: Asymptotic Periodicity of Markov and Related Operators. Dynamics Reported, Expositions in Dynamical Systems, pp. 31-68. Springer, Berlin (1993)

14. Komornik, J., Lasota, A.: Asymptotic decomposition of Markov operators. Bull. Polish. Acad. Sci. Math. 35(5-6), 321-327 (1987)

15. Krengel, U., Lin, M.: On the deterministic and asymptotic $\sigma$-algebras of a Markov operator. Canad. Math. Bull. 32(1), 64-73 (1989)

16. Lasota, A.: Asymptotic behaviour of solutions: statistical stability and chaos. In: Proceedings of the ICM '82. PWN/North Holland (1984)

17. Lasota, A., Li, T.Y., Yorke, J.A.: Asymptotic periodicity of the iterates of Markov operators. Trans. Am. Math. Soc. 286(2), 751-764 (1984)

18. Lasota, A., Mackey, M.C.: Chaos, Fractals and Noise: Stochastic Aspects of Dynamics. Springer, New York (1993)

19. Lasota, A.: Układy dynamiczne na miarach, Wydawnictwo Uniwersytetu Ślaskiego (2008)

20. Miklavčič, M.: Asymptotic periodicity of the iterates of positivity preserving operators. Trans. Am. Math. Soc. 307, 469-490 (1988) 
21. Schaefer, H.H.: Banach Lattices and Positive Operators. Springer, New York (1974)

22. Sine, R.: Constricted systems. Rocky Mt. J. Math. 21(4), 1373-1383 (1991)

23. Zaharopol, R.: Invariant Probabilities of Transition Functions. Springer, Berlin (2014)

Publisher's Note Springer Nature remains neutral with regard to jurisdictional claims in published maps and institutional affiliations. 